\title{
openheart A new electrode placement method for obtaining 12-lead ECGs
}

\author{
Gabriel M Khan
}

To cite: Khan GM. A new electrode placement method for obtaining 12-lead ECGs. Open Heart 2015;2:e000226. doi:10.1136/openhrt-2014000226

\footnotetext{
- Additional material is available. To view please visit the journal (http://dx.doi.org/ 10.1136/openhrt-2014000226)
}

Received 2 December 2014 Revised 17 April 2015 Accepted 6 May 2015

CrossMark

Dr Gabriel M Khan MB, BCh, MD (Queen's Belfast), FRCP (London) FRCP C, FACP, FACC, Associate Professor, Department of Medicine and Division of Cardiology, University of Ottawa, The Ottawa Hospital, Ottawa, Ontario, Canada

Correspondence to Dr Gabriel M Khan; DrKhanBooks@gmail.com

\section{ABSTRACT}

Objective: Worldwide, more than 50 million ECGs are carried out annually. Studies show that the standard wrist-ankle placement method may produce poor quality ECGs. Factors including limb movement, tremor, anxiety, cold extremities cause artefacts (distortions of the baseline and ECG waveforms). A problem exists. Physicians face a challenge interpreting poor quality ECGs. Diagnostic delays occur. Patients are subjected to recalls; compassion initiated this study. Torso (modified) leads are favoured as they provide rapid acquisition of better quality ECGs, but cause erroneous electrocardiography. Investigators have not attempted to correct these errors. This study analyses the errors and aims to rectify them.

Methods: Our pilot and other studies indicated that torso electrodes placed close to the heart cause an increase in R wave amplitude $>3 \mathrm{~mm}$ in inferior leads that results in disappearance of inferior infarcts; a decrease $>3 \mathrm{~mm}$ in 1 and aVL may cause false lateral infarcts. We hypothesised that finding electrode placements that do not cause alterations in $\mathrm{R}$ wave amplitude should correct these problems. Several electrode placements were assessed to obtain ECGs identical with the standard, but with better quality. A total of 1112 patients received standard and new electrode placement recordings. ECG parameters were assessed. A blinded interpreter assisted.

Results: Electrodes positioned on the mid-arm and lower abdomen revealed ECGs identical with the standard without artefacts or loss of inferior or appearance of lateral infarcts.

Conclusions: This study proved that the new method is faultless and provides better quality ECGs, no recalls and without risk for misinterpretations. Not having to remove leg garments is convenient and allows more rapid acquisition of ECGs. The forearms are freed for, intravenous, radial access, and ECGs needed during procedures. The findings have implications worldwide for patients, clinicians and technicians, and generate the need to study the method in emergency settings.

\section{INTRODUCTION}

For more than 65 years, the standard 12-lead ECG has been obtained by placing electrodes on the ankles and wrists, along with chest leads ${ }^{1}$ and it is well established that limb movement, subtle muscle tremor and interference from muscle potential results in

\section{KEY MESSAGES}

What is already known about this subject?

- It is well established that the Standard 12-lead ECG obtained using the wrist and ankle placement is subject to artefacts (distortions) of the baseline and ECG wave forms caused by limb movement and interference from muscle potential, resulting in poor quality ECGs and thus, the unfortunate recall of patients. Physicians face a major challenge interpreting poor quality ECGs.

- Torso (modified) leads are favoured as they provide rapid acquisition of better quality ECGs.

- Inconvenience of removing hosiery or other leg garments is avoided. However, torso leads cause disappearance of inferior infarcts and the appearance of false lateral infarcts that disqualifies modified leads as a standard recording.

What does this study add?

- A solution for torso (modified) leads. This study tested a partial torso lead system that proved the new electrode placement method can replace the wrist-ankle placements for routine electrocardiography.

- Better quality ECGs allow for more precise interpretation, less diagnostic delays, with no recall of patients.

- Compassion for the elderly and infirm who are recalled for repeat ECGs.

How might this impact on clinical practice?

- The ECG, a frequently used diagnostic test, is made more reliable. Physicians can provide more precise interpretation for all ECGs. Patients obtain better care.

- The findings generate a reason to initiate studies in emergency settings where lives can be saved worldwide by more rapid acquisition of better quality ECGs and diagnosis that is needed for timely percutaneous coronary intervention or thrombolysis.'

artefacts $^{2}{ }^{3}$ that alter interpretation. Torso (modified) leads have been shown to abolish such artefacts ${ }^{23}$ but produce erroneous ECG that disqualifies modified leads as a standard recording. A methodological question remains unresolved. Could modification of the electrode placements used in studies of 
modified leads prevent the errors observed? Sound methodology to rectify the problems caused by torso leads required a thorough review and understanding of relevant study findings accompanied by a pilot study.

- Takuma $e t a l^{2}$ in a study of 30 patients, placed electrodes on the shoulder tip and the anterior superior iliac spine to permit easier and more rapid ECG acquisition in emergency settings and obtain higher quality recordings. The electrode placements on the acromion process, and near the iliac crest caused abnormal changes in $\mathrm{R}$ wave amplitude. The investigators obtained better quality ECGs and emphasised the advantages of modified leads, but did not recommend them for routine use. ${ }^{2}$

- Jowett $e t a l^{3}$ indicated that 'The appeal of this easily applied lead system has resulted in extensive use in our hospital, both in emergency and non-emergency situations'. ${ }^{3}$ Their study of 100 patients resulted in the disappearance of five of six inferior infarcts that were previously observed on the standard ECG. ${ }^{3}$ The electrode sites were as performed by Takuma et al. ${ }^{2}$ The ECGs of $43 \%$ of patients showed movement artefact with wrist-ankle placement compared with no artefacts with torso recordings. $\mathrm{R}$ wave amplitude was altered.

Papouchado et at $t^{4}$ assessed the differences between the standard 12-lead ECG and the modified (Mason and Likar $^{5}$ ) exercise lead system in 29 patients. This showed that when the lower limb electrode was positioned in the nipple line at the level of the umbilicus, and moved vertically to the costal margin or higher, a marked increase in $\mathrm{R}$ wave amplitude occurred in inferior leads and $\mathrm{a}$ decrease in $\mathrm{R}$ wave amplitude was observed in lead 1 and aVL occurred. Vertical but not horizontal abdominal electrode placements were assessed. ${ }^{4}$ Most important, abdominal, horizontal variations were assessed in the present study.

Wilson ${ }^{6}$ discussing the standard limb leads placed on the chest stated "the principles of the equilateral triangle $^{7}$ should not be applied to any system of chest leads whatsoever". ${ }^{6}$ Wilson emphasised that since all points on the arm have the same potential, it does not matter whether the electrode is placed on the wrist, the forearm or the upper arm; the arm acts mainly as an extension of the wire attached to it. ${ }^{6}$

\section{METHODS}

The acromion process is part of the scapular and thus, part of the trunk. It is not surprising therefore, that this torso electrode position used by Jowett et $a l^{3}$ caused the disappearance of five of six inferior infarcts and appearance of false lateral infarcts and showed significant variation of waveforms in the study by Takuma et $a l^{2}{ }^{2}$ The present study avoided this erroneous electrode placement. Findings from our pilot study, and data from investigators ${ }^{2-4} 6$ initiated the following thinking and deductions to design an appropriate method to resolve the problems attached to torso (modified) leads.

1. The problem that surrounds torso placements is an increase in $\mathrm{R}$ wave amplitude $>2 \mathrm{~mm}$ in inferior leads that can result in disappearance of inferior myocardial infarction (MI), and a $>2 \mathrm{~mm}$ decrease in leads 1 and aVL that may simulate a lateral infarct. Right axis change is caused by alterations in $\mathrm{R}$ wave amplitude.

2. Finding electrode placements that do not cause alterations in $\mathrm{R}$ wave amplitude should correct the problems.

3. The electrodes for the limb leads must not all be placed on the torso, because this invalidates Einthoven's ${ }^{7}$ theory of the equilateral triangle. ${ }^{6}$

4. Electrodes must not be positioned on the acromion.

5. The upper limb electrode must be placed on the arm at any point ${ }^{6}$ but must be below the shoulder joint. This study used the mid-arm; thus, the new electrode placement (NEP) cannot be described as torso leads.

The new electrode placements must prevent the following errors observed with torso leads:

- An unfavourable change in $\mathrm{R}$ wave amplitude in the limb leads that causes:

- The disappearance of inferior MI;

- The appearance of QS in leads 1 and aVL, causing a false lateral infarct;

- Right axis deviation.

Our pilot study placed electrodes on the mid-arm, various abdominal sites and infraclavicular. Finally, the lower limb electrodes were moved to the lower abdomen $7.6 \mathrm{~cm}$ ( 3 inches) below a horizontal line running across the umbilicus. This position, well below the umbilicus, was maintained for all 1112 patients tested (group A, B, C).

Thought was given to performing ECGs simultaneously, but it was decided that comparing a standard ECG with an NEP ECG performed $1 \mathrm{~min}$ apart would not change the interpretation except for arrhythmias. Special software provided duplicate ECGs to be performed at nine laboratory locations, which allowed two electronic ECGs to reach the primary investigator for interpretation. The interpreter was not blinded for the electrodes. EGCs from 1112 patients were assessed. Patients were allocated to group A (786 patients) including 30 with inferior MI, group B (50 patients) and group C (276 patients) including 10 patients with inferior MI.

Group A: Enrollment of patients age 33-87 was started following ethics approval, 3 February 2014 in the city of Ottawa. A consent form was signed. Patients for NEP recordings had the ankle electrodes placed $7.6 \mathrm{~cm}$ below the umbilicus and $10 \mathrm{~cm}$ on either side of the umbilical midline. 651 patients attending Gamma Dynacare laboratories (GDML) for routine ECGs had a standard 12-lead ECG completed; and within $1 \mathrm{~min}$, an NEP ECG was also carried out. A total of 135 patients seen in a cardiology referral centre had duplicate ECGs performed, and were supervised by the primary investigator (figure 1). 
Figure 1 The arm electrodes ( $A$ and $B$ ) are placed on the mid-arm, on the lateral aspect of the biceps, immediately below the V4 horizontal line. The abdominal electrodes (C and D) placed $7.6 \mathrm{~cm}$ ( $\sim 3$ inches) below the umbilical horizontal line, and $5 \mathrm{~cm}$ ( $\sim 2$ inches) on either side of the umbilical vertical line. The distance between these two electrodes to be $10 \mathrm{~cm}$ ( 4 inches).

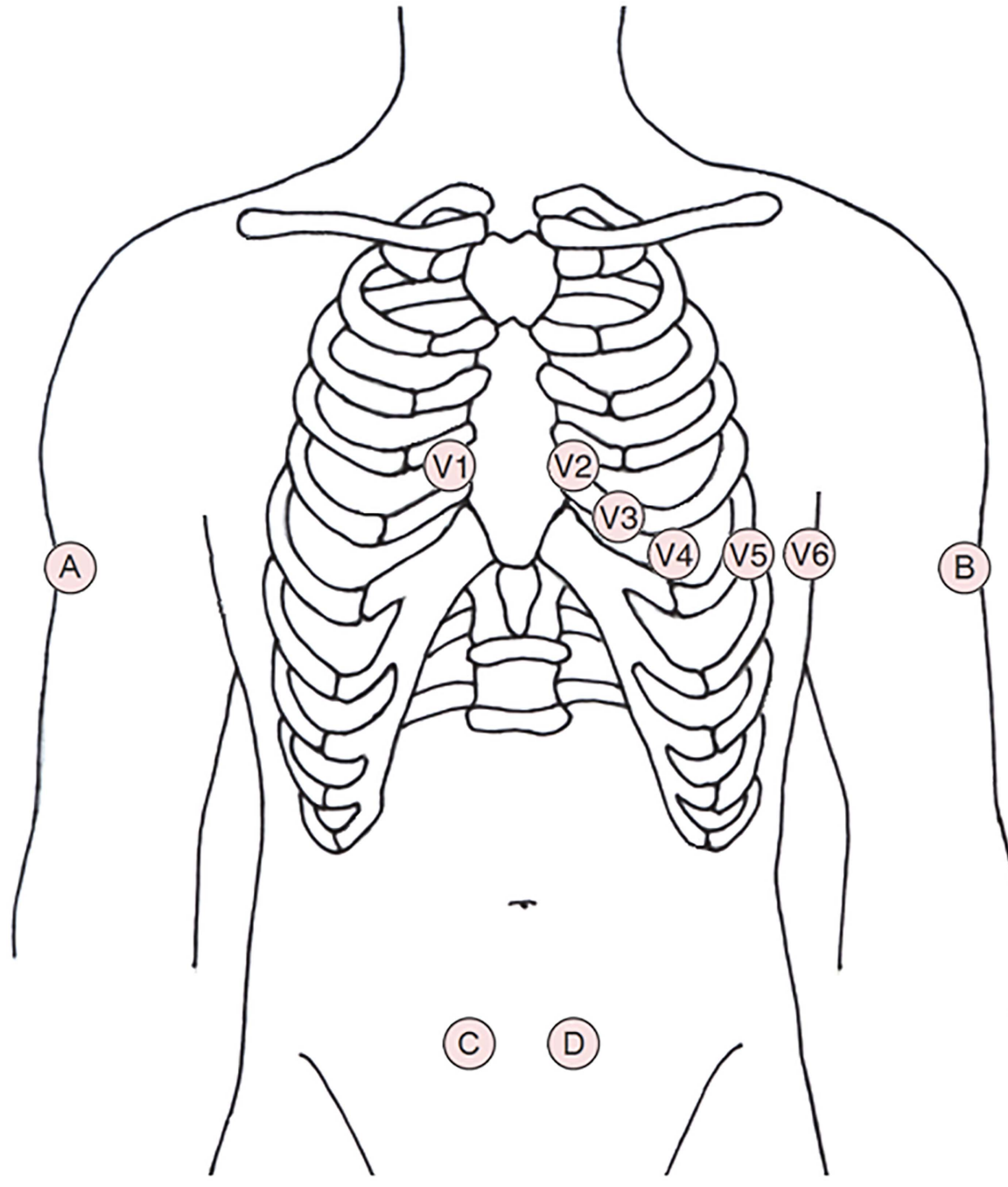

4. Artefacts-recalls because of artefacts were assessed and poor quality noted.

\section{RESULTS}

Figures 2-5 show that the five major problems observed with torso leads are not observed with recordings carried out using the new placements.

Group A

1. Artefacts-figure 2A shows a standard ECG with artefacts that disappear on the NEP tracing figure 2B (see online supplementary file appendix 3, eFigure 23)

Similar or less prominent artefacts on standard 12-lead ECGs were responsible for $19(2.94 \%)$ recalls of 651 patients but no recalls were required for NEP ECGs. The majority of patient ECGs that revealed major artefacts exhibited clearing of most artefacts when NEP ECGs were completed. Poor quality ECGs were observed with STD ECGs in $\sim 10 \%$ of all cases versus $\sim<2 \%$ for NEP ECGs.

2. Inferior infarcts-duplicate ECGs from 30 group A patients with proven inferior MI revealed no differences and were reassessed by a blinded interpreter who agreed with our findings. Figure 3 shows the ECG of a patient with a proven old inferior infarct. Diagnostic wide $Q$ 
Figure 2 (A) Standard 12-lead ECG showing artefacts (see online supplementary file appendix 3, eFigure 23). (B). New electrode placement ECG, carried out within $30 \mathrm{~s}$ of the standard tracing reveals no artefacts (see online supplementary file appendix 3, eFigure 24).
A

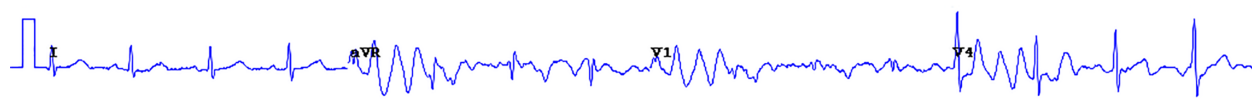

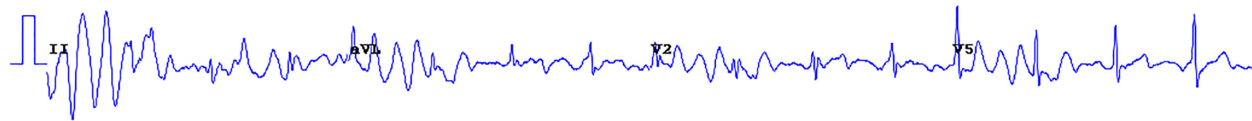

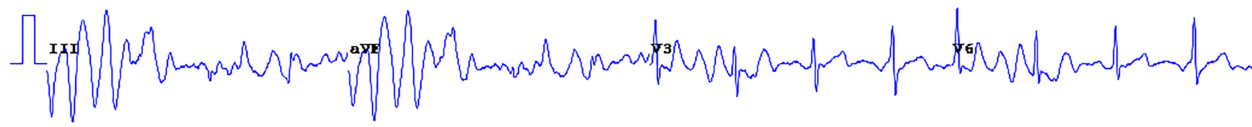

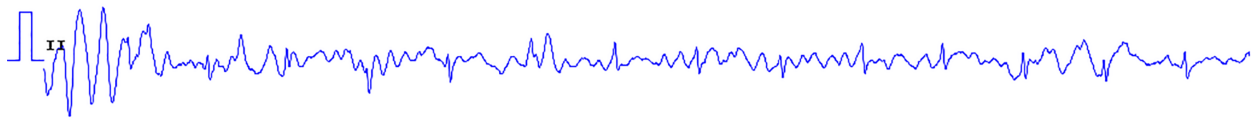
$25 \mathrm{~mm} / \mathrm{sec} 10 \mathrm{~mm} / \mathrm{mV}[0.5-35] \mathrm{Hz}-60 \mathrm{~Hz}$

B

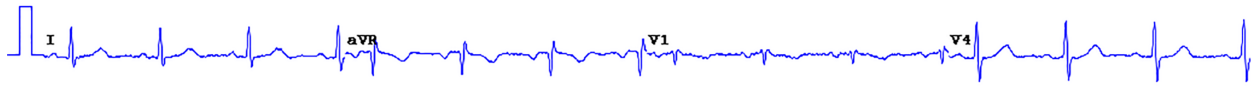

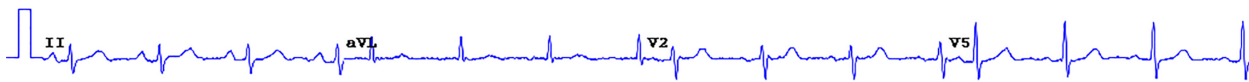

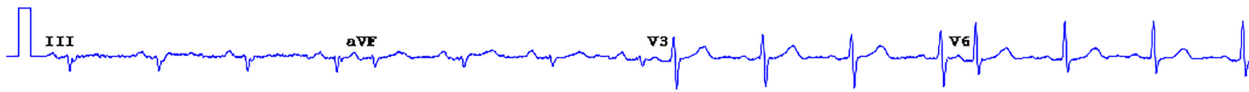

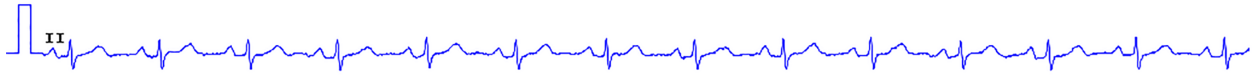

$25 \mathrm{~mm} / \mathrm{sec} 10 \mathrm{~mm} / \mathrm{mV}[0.5-35] \mathrm{Hz} \sim 60 \mathrm{~Hz}$ waves are seen in leads 11, 111, aVF: (a) standard ECG, (b) NEP ECG. The NEP ECG of a 57-year-old female patient, with acute chest pain and acute inferior MI, is shown in figure 4. No standard ECG was performed, in order to avoid delays to percutaneous coronary intervention (PCI).

3. Decrease in $R$ wave amplitude in 1 and $a V L-N o$ significant decrease in $\mathrm{R}$ wave amplitude (equal to or $>2 \mathrm{~mm}$ ) was observed in any ECG from group A (786 patients), and no QS pattern was observed in leads 1 and aVL. Figure 5A shows a standard ECG tracing with low amplitude $(<3 \mathrm{~mm}) \mathrm{R}$ wave in lead 1 and aVL. Figure 5B gives the NEP ECG showing no appreciable changes. No QS pattern, in leads 1 and aVL, false lateral infarct pattern, was observed from the screening of all patient ECGs.

4. $R$ wave amplitude changes in $V$ leads: no changes were observed, and no differences in ECGs were noted in patients with right and left bundle branch block, left ventricular hypertrophy or anterior infarction.

5. Right axis deviation-fifty group A patients showed a non-significant axis change of zero to $7^{\circ}$
Group B-As demonstrated by the pilot study, with abdominal electrode placements as follows: $7.6 \mathrm{~cm}$ beneath the umbilicus and horizontally, close to the iliac crest, $20 \mathrm{~cm}$ or more from the umbilicus, caused a 1$2 \mathrm{~mm}$ change in $\mathrm{R}$ wave amplitude versus no change with electrodes $10 \mathrm{~cm}$ from the umbilicus. Electrode placements $10 \mathrm{~cm}$ from the umbilicus produced recordings that were virtually the same as the standard, and was used successfully in all 786 group A patients. It was felt necessary however, to assess electrodes placed $5 \mathrm{~cm}$ from the umbilicus (figure 1). Therefore, on completion of group A, 50 patients (group B) were tested with triplicate ECGs, using placements $10 \mathrm{~cm}$ and $5 \mathrm{~cm}$ from the umbilicus compared with standard recordings. For each sets of recordings, the technician was supervised by the investigator.

Results Group B: the abdominal placement $10 \mathrm{~cm}$ from the umbilicus showed ECGs virtually identical with the standard in all patients. The $\mathrm{R}$ wave amplitude was equal to the standard in 44 of 50 patients. In six patients minimal, $0.5-1 \mathrm{~mm}$ non-significant $\mathrm{R}$ wave changes were observed. Electrode placement $5 \mathrm{~cm}$ form the umbilicus 
A

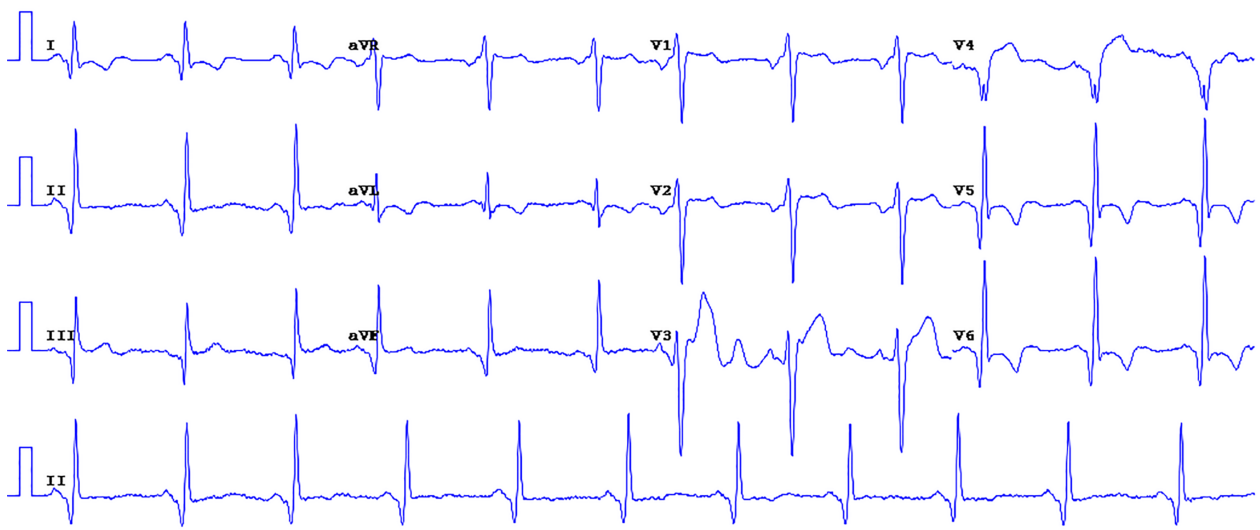

$25 \mathrm{~mm} / \mathrm{sec} 10 \mathrm{~mm} / \mathrm{mV}[0.5-35] \mathrm{Hz} \sim 60 \mathrm{~Hz}$

B

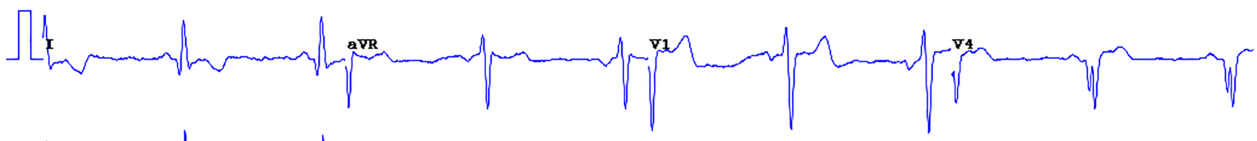

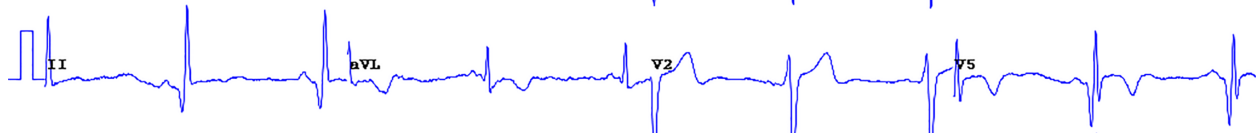

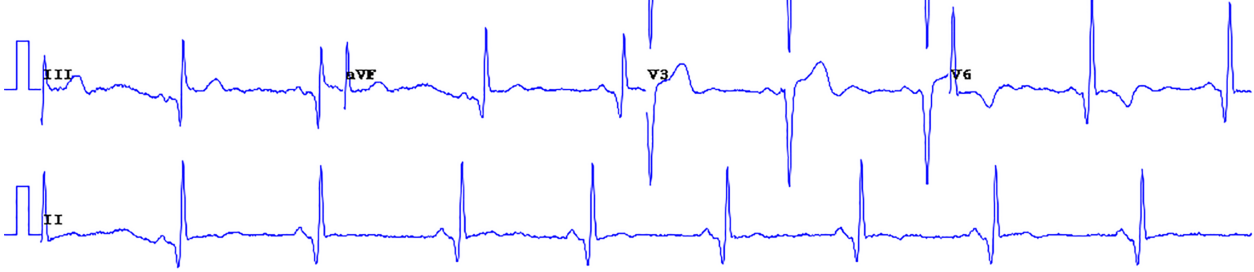

$25 \mathrm{~mm} / \mathrm{sec} 10 \mathrm{~mm} / \mathrm{mV}[0.5-35] \mathrm{Hz} \sim 60 \mathrm{~Hz}$

Figure 3 (A) Standard lead ECG: deep wide $Q$ waves in leads 11, 111, aVF: old inferior, and anterolateral myocardial infarction, artefacts in V3, V4 (see online supplementary file, appendix 1, eFigures 1-59, eAppendix 3, figures 1-20). (B) New electrode placement ECG, old inferolateral infarct; the tracing is similar to the standard recording, but with clearing of artefacts in V3 and V4. The R wave amplitude in all 12 leads is the same in the standard and NEP ECG.

(figure 1) revealed identical wave forms and $\mathrm{R}$ wave amplitude as the standard in all 50. Although $\mathrm{R}$ wave amplitude changes at $10 \mathrm{~cm}$ from the umbilicus were considered not significant, and was used successfully in all group A patients, group $\mathrm{C}$ was started with abdominal placement $5 \mathrm{~cm}$ from the umbilicus (figure 1). Group B patients were, in essence, a second pilot study and were not counted in the statistical analysis.

Group C-276 patients attending GDML had duplicate ECGs performed with electrode placements as shown in figure 1.

Results The principal investigator, and a blinded interpreter compared NEP and standard ECGs and found no differences in wave forms, or parameters. Table 1 gives ECG parameters in .30 consecutive patients. No changes in $\mathrm{R}$ wave amplitude were noted, and no false lateral infarcts appeared. The ECGs of 10 patients with proven old inferior MIs revealed the same waveforms, with no disappearance of inferior infarcts (see online supplementary file appendix 3). $\mathrm{P}$ waves, ST segment and $\mathrm{T}$ waves were identical with that of the standard ECG. No ECG differences were noted in patients with left and right bundle branch block, atrial fibrillation or premature beats. Arrhythmia diagnosis was unchanged by the new method. No R wave amplitude changes occurred in leads 1 and aVL, inferior or chest leads. Forty-six patients had low amplitude $\mathrm{R}$ waves $<5 \mathrm{~mm}$ in leads 1 and aVL, and 16 with the lowest $\mathrm{R}$ wave amplitudes $<4 \mathrm{~mm}$ were chosen to test the new placement method for flaws. No occurrence of a QS pattern in leads 1 and aVL or false lateral infarct was noted in the 16 test patients ECGs (see test cases in the online supplementary file, appendix 2, eFigures 1 to 32 and appendix 3 eFigure 21, 22).

A blinded interpreter assessed:

- All 276 group C patients. 

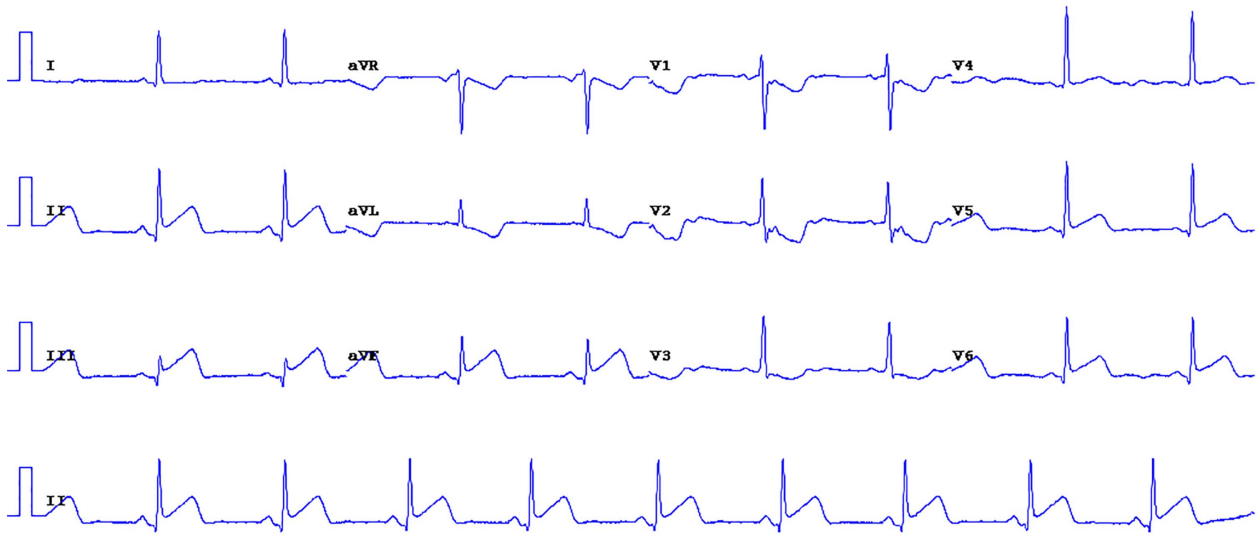

$25 \mathrm{~mm} / \mathrm{sec} 10 \mathrm{~mm} / \mathrm{mV}[0.5-35] \mathrm{Hz} \sim 60 \mathrm{~Hz}$

Figure 4 New electrode placement ECG from a female, age 57 with acute chest pain, shows abnormal ST elevation in leads $11,111, a V F$, V5, V6: typical findings of acute inferior MI with lateral involvement, ST segment elevation MI. Reciprocal depression V1-V2, aVL is not diagnostic but helps confirm the diagnosis of acute MI.

Figure 5 (A) Standard 12-lead ECG: low $R$ wave amplitude in lead 1 , aVL; a test case chosen to flaw the new electrode placement (see online supplementary file, appendix 2 , eFigures 1 to 32 and appendix 3 eFigure 21, 22). (B) New electrode placement ECG; similar to that of the standard ECG. The $R$ wave amplitude in all 12 leads is virtually the same. The very small $R$ wave in $\mathrm{aVL}$ did not change to a QS pattern (see online supplementary file eAppendix 2, eFigures 1 to 32).
A
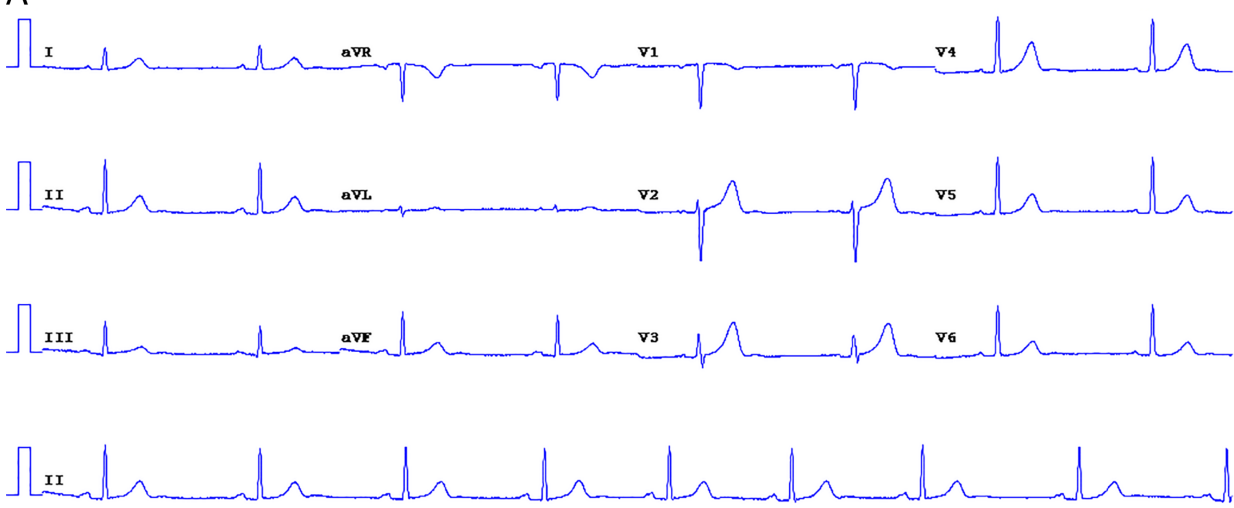

$25 \mathrm{~mm} / \mathrm{sec} 10 \mathrm{~mm} / \mathrm{mV}[0.5-35]$ Hz $\sim 60 \mathrm{~Hz}$

B
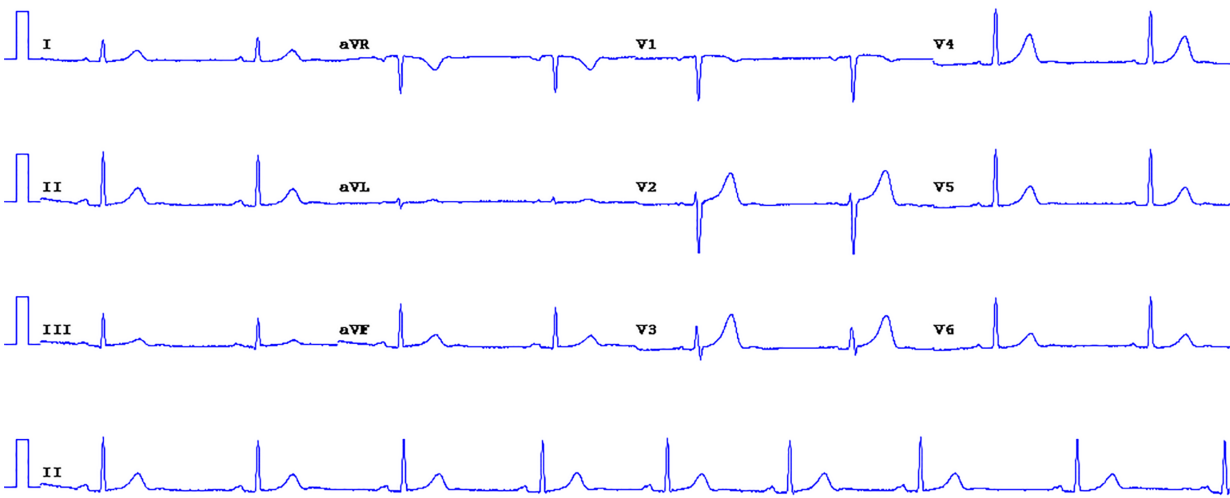

$25 \mathrm{~mm} / \mathrm{sec} 10 \mathrm{~mm} / \mathrm{mV}[0.5-35] \mathrm{Hz} \sim 60 \mathrm{~Hz}$ 
Table $1 \mathrm{R}$ wave amplitude in lead 1 and ECG parameters in 30 patients

\section{R wave amplitude and ECG parameters in 30 patients from group C (276 patients)}

\begin{tabular}{|c|c|c|c|c|c|c|c|c|c|c|}
\hline \multirow{2}{*}{$\begin{array}{l}\text { Subject } \\
\text { Parameters/ECG type }\end{array}$} & \multicolumn{2}{|l|}{1} & \multicolumn{2}{|l|}{2} & \multicolumn{2}{|l|}{3} & \multicolumn{2}{|l|}{4} & \multicolumn{2}{|l|}{5} \\
\hline & NEP & STD & NEP & STD & NEP & STD & NEP & STD & NEP & STD \\
\hline PR interval (ms) & 177 & 178 & 152 & 155 & 172 & 173 & 173 & 172 & 148 & 150 \\
\hline QRS duration & 98 & 95 & 98 & 100 & 110 & 112 & 105 & 101 & 100 & 92 \\
\hline Axis & -17 & -19 & -3 & -7 & -31 & -27 & -11 & -8 & -38 & -33 \\
\hline $\mathrm{R}$ wave amplitude lead $1(\mathrm{~mm})$ & 14 & 14 & 10 & 11 & 6 & 5 & 13 & 15 & 6 & 6 \\
\hline QTC & 404 & 402 & 438 & 424 & 443 & 443 & 400 & 406 & 435 & 427 \\
\hline
\end{tabular}

\begin{tabular}{|c|c|c|c|c|c|c|c|c|c|c|}
\hline Subject & 6 & & 7 & & 8 & & 9 & & 10 & \\
\hline PR interval (ms) & 200 & 200 & 130 & 128 & 158 & 148 & 170 & 168 & 173 & 170 \\
\hline QRS duration & 132 & 133 & 82 & 87 & 67 & 67 & 110 & 115 & 129 & 132 \\
\hline Axis & -83 & -84 & 55 & 48 & 83 & 82 & 65 & 67 & 89 & 91 \\
\hline $\mathrm{R}$ wave amplitude lead $1(\mathrm{~mm})$ & 3 & 3.5 & 7 & 7 & 5 & 5 & 5 & 5 & 4 & 4 \\
\hline QTC & 432 & 430 & 392 & 398 & 382 & 385 & 444 & 442 & 452 & 448 \\
\hline
\end{tabular}

\begin{tabular}{|c|c|c|c|c|c|c|c|c|c|c|}
\hline Subject & 11 & & 12 & & 13 & & 14 & & 15 & \\
\hline PR interval (ms) & 158 & 160 & 225 & 227 & 203 & 197 & 150 & 143 & 173 & 177 \\
\hline QRS duration & 84 & 83 & 175 & 173 & 120 & 135 & 93 & 97 & 180 & 190 \\
\hline Axis & 4 & 14 & -65 & -63 & -41 & -40 & 6 & 4 & 82 & 81 \\
\hline$R$ wave amplitude lead $1(\mathrm{~mm})$ & 10 & 10 & 3 & 3 & 11 & 11 & 7 & 7 & 3 & 3 \\
\hline QTC & 402 & 403 & 496 & 482 & 425 & 452 & 396 & 399 & 499 & 505 \\
\hline Subject & 16 & & 17 & & 18 & & 19 & & 20 & \\
\hline PR interval (ms) & 150 & 153 & 168 & 155 & 130 & 133 & 155 & 153 & 193 & 187 \\
\hline QRS duration & 97 & 97 & 105 & 118 & 183 & 197 & 85 & 84 & 114 & 113 \\
\hline Axis & 36 & 39 & -13 & -15 & 13 & 6 & 68 & 64 & 62 & 55 \\
\hline R wave amplitude lead $1(\mathrm{~mm})$ & 7 & 6 & 7 & 7 & 13 & 13 & 4 & 4.5 & 6.5 & 7 \\
\hline QTc & 400 & 409 & 419 & 424 & 499 & 493 & 412 & 411 & 420 & 425 \\
\hline
\end{tabular}

\begin{tabular}{|c|c|c|c|c|c|c|c|c|c|c|}
\hline Subject & 21 & & 22 & & 23 & & 24 & & 25 & \\
\hline PR interval (ms) & 187 & 188 & 143 & 140 & 158 & 162 & 147 & 137 & 187 & 210 \\
\hline QRS duration & 130 & 129 & 97 & 100 & 87 & 85 & 130 & 140 & 93 & 97 \\
\hline Axis & -24 & -25 & 25 & 22 & 39 & 40 & -54 & -49 & -24 & -21 \\
\hline R wave amplitude lead $1(\mathrm{~mm})$ & 10 & 9 & 9 & 9 & 5 & 5 & 7 & 7 & 7 & 7 \\
\hline QTc & 418 & 409 & 406 & 435 & 433 & 436 & 448 & 445 & 434 & 140 \\
\hline
\end{tabular}

\begin{tabular}{lrrrrrrrrrr}
\hline Subject & \multicolumn{1}{c}{$\mathbf{2 6}$} & \multicolumn{1}{c}{$\mathbf{2 7}$} & & $\mathbf{2 8}$ & \multicolumn{2}{c}{$\mathbf{2 9}$} & \multicolumn{3}{c}{$\mathbf{3 0}$} \\
\hline PR interval (ms) & 183 & 180 & 173 & 173 & 157 & 147 & 150 & 147 & 223 & 220 \\
QRS duration & 87 & 90 & 124 & 114 & 76 & 86 & 93 & 86 & 90 & 90 \\
Axis & 68 & 68 & 40 & 44 & 23 & 18 & 51 & 50 & 37 & 34 \\
R wave amplitude lead 1 (mm) & 4 & 4 & 3 & 3 & 8 & 8 & 7 & 7.5 & 6.5 & 6.5 \\
QTc & 430 & 411 & 434 & 434 & 385 & 392 & 431 & 432 & 400 & 408 \\
\hline
\end{tabular}

- All ECGs that are in the online supplementary files.

- 10 duplicate ECGs with inferior MIs (see online supplementary file appendix 3).

- The QRS axis in 50 consecutive group $\mathrm{C}$ patients (see online supplementary file table S2).

- 16 duplicate ECGs, test cases showing very low R waves in leads 1 and aVL for QS pattern or false lateral MI (see online supplementary file eAppendix 2, eFigures 1 to 32).

- 30 ECGs showing old inferior MIs (see online supplementary file eAppendix 1).
Figure 6A shows the standard 12-lead ECG from a patient with Eisenmenger syndrome, marked right ventricular hypertrophy: $\mathrm{R}>1 \mathrm{mV}$ in $\mathrm{V} 1, \mathrm{R} / \mathrm{S}>1$ in $\mathrm{V} 1, \mathrm{R} / \mathrm{S}$ $<1$ in $\mathrm{V} 4, \mathrm{~V} 5, \mathrm{~V} 6$, right atrial hypertrophy and right axis deviation 153. The NEP ECG shown in figure $6 \mathrm{~B}$ reveals virtually the same waveforms and QRS axis of 155 .

\section{STATISTICAL ANALYSIS}

Intraclass correlation (ICC) estimates were calculated in order to quantify the agreement between the NEP ECG 
and the standard (STD) ECG with respect to $\mathrm{R}$ wave amplitude in Lead 1 and the different ECG parameters measured in $n=30$ patients. The NEP and STD method have a high level of agreement in measurement of the $\mathrm{R}$ wave amplitude in Lead 1, ICG correlation 0.984 . Table 1 shows $\mathrm{R}$ wave amplitudes in Lead 1, and ECG parameters for the NEP and standard ECGs in 30 patients. See table 2 for ICC estimates and Spearman correlations. Spearman and Pearson correlations as well as the ICC were also calculated in order to quantify the agreement in the axis deviation measured in 50 consecutive patients (See table 2A, table 1 in the supplement gives actual measurements of axis deviation).

When comparing diagnosis with STD and NEP, sensitivities and specificities were also calculated along with exact binomial 95\% CIs (table 3). The NEP and standard ECG recordings from 40 patients with inferior infarction and 149 patients with normal ECGs show similar sensitivities and specificities (table 3).

\section{DISCUSSION}

This large study proved conclusively that the NEP method is sound and suitable for routine ECG. Five small studies compared standard limb lead placements and torso placed electrodes. Diamond $e t a l^{8}$ studied only 11 patients using all torso leads. Variations in QRS amplitude were observed but reported as not significant. The investigators concluded that the torso lead system appears to produce an ECG recording interchangeable with the standard limb placement. Time has shown that this conclusion is incorrect. Importantly, there were no patients with inferior infarction in the study. The standard ECG in one patient "with a marked tremor was interpretable only with great difficulty; the torso system gave a far superior ECG'.

A 1989 study by Sevilla et at assessed 44 patients, using exercise torso-lead placements to acquire 12-lead ECGs. The wrist electrodes were placed under the clavicle in the mid-clavicular lines. The ankle electrodes

\begin{tabular}{|c|c|c|c|}
\hline & $\begin{array}{l}\text { ICC } \\
\text { intraclass } \\
\text { correlation }\end{array}$ & Pearson & Spearman \\
\hline \multicolumn{4}{|c|}{ ECG parameters ( $n=30$ patients) } \\
\hline PR interval & 0.965 & & 0.967 \\
\hline QRS duration & 0.977 & & 0.953 \\
\hline QRS axis & 0.996 & & 0.996 \\
\hline $\begin{array}{l}\mathrm{R} \text { wave } \\
\text { amplitude lead } 1\end{array}$ & 0.984 & & 0.982 \\
\hline QTc & 0.943 & & 0.899 \\
\hline $\begin{array}{l}\text { Axis deviation } \\
\text { ( } n=50 \text { consecutive } \\
\text { patients) }\end{array}$ & 0.992 & 0.993 & 0.990 \\
\hline
\end{tabular}

were positioned in the anterior axillary lines above the costal margins. Loss of ECG inferior pattern occurred in 11 of 16; a false lateral infarct was seen in $19 \%$ of patients and a significant change in frontal axis was noted.

Pahlm et $a l^{10}$ reported on torso placements in 30 patients. Simultaneous recordings revealed significant frontal plane axis shift and diminished Q-wave durations in lead aVF compared with those of the standard.

The 1995 emergency room study of 30 patients with only one inferior infarct by Takuma et $a l^{2}$ showed minor QRS amplitude changes, and was not recommended for routine use. However, the quality of recordings were definitely improved. ${ }^{2}$ The wrist electrodes were placed on the shoulder tip, the acromion process.

The 2005, ECG study of 100 patients by Jowett $e t a l^{3}$ with torso electrode similar to the Takuma placement resulted in loss of five of six inferior infarcts, one false lateral infarct, and important amplitude changes that created eight possible myocardial infarcts to appear. A marked change in axis was observed.

Importantly, these investigators ${ }^{2} 3910$ did not attempt to correct the problems caused by torso leads. The present study analysed the problems and rectified them.

The 2007 AHA/ACG guidelines on ECG Part 1: recommends studies to look at modified leads. ${ }^{11}$ Previous studies ${ }^{3} 49$ and our pilot study indicate that modified leads (all torso) cause serious errors.

The present study shows that a lower torso and upper arm placement is reliable. No inferior infarcts were lost and no false lateral infarcts were produced by the NEP. If the abdominal electrode placement is as shown in figure 1 , the NEP does not cause a change in $\mathrm{R}$ wave amplitude. Thus, disappearance of inferior MI and causation of a lateral infarct cannot occur. Each ECG should be labelled 'new electrode placement' to distinguish the system from modified leads that provide erroneous ECG recordings. However, a $5 \mathrm{~cm}$ lateral displacement of the abdominal electrodes should not alter interpretation as shown for all 786 group A patients including 30 patients with inferior infarcts in whom the abdominal electrode placement was $\sim 5 \mathrm{~cm}$ lateral to that used for group $\mathrm{C}$ (see figure 1).

\section{Limitations and further studies}

This study was not performed in emergency rooms, but the NEP ECG captured the ST-elevation, reciprocal depression and $Q$ waves of acute inferior MI remarkably well (figure 4). Owing to the rapidity of application, and better quality ECGs, the NEP should prove useful for diagnosis in patients with probable acute coronary syndrome and in emergency settings. The NEP needs to be studied in this population of patients.

\section{CONCLUSION}

This large study showed that the new method produces ECGs that are identical to the standard and provides 
A

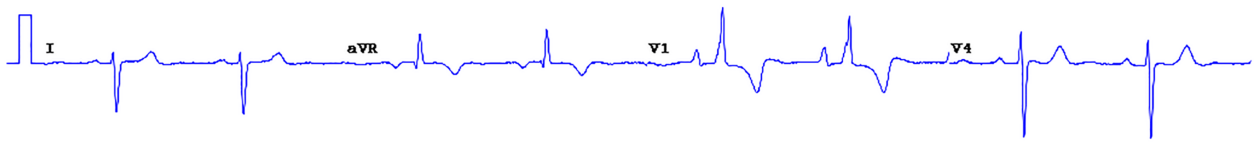

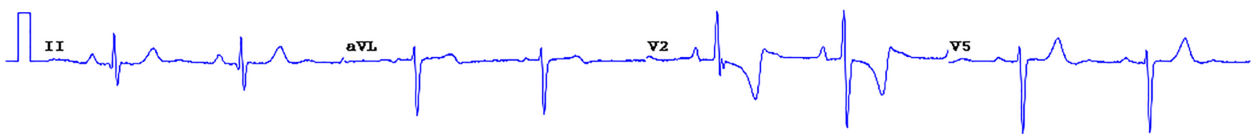

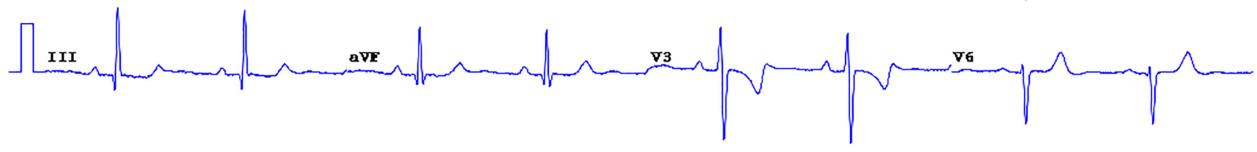

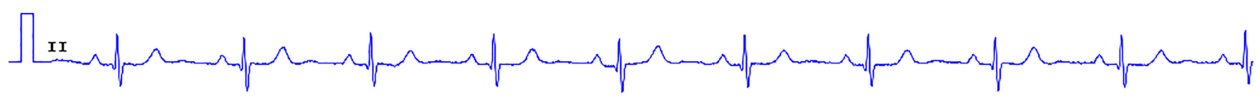

$25 \mathrm{~mm} / \mathrm{sec} 10 \mathrm{~mm} / \mathrm{mV}[0.5-35]$ Hz $-60 \mathrm{~Hz}$

B

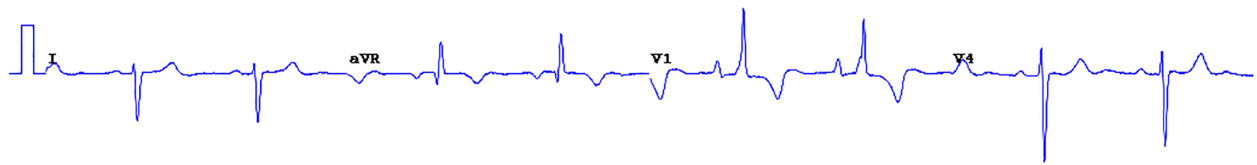

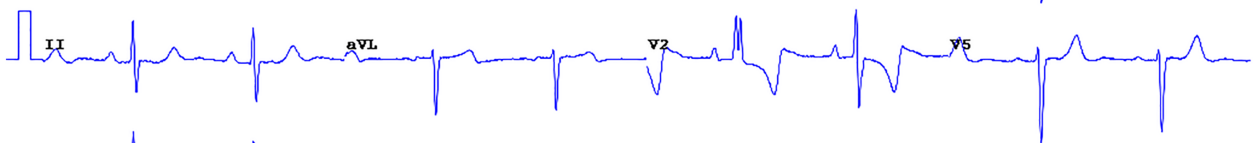

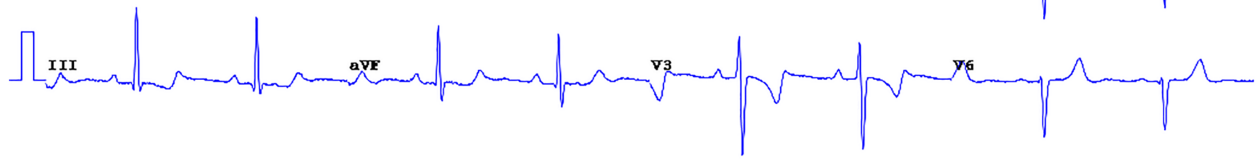

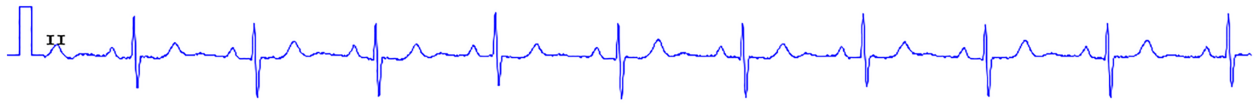

$25 \mathrm{~mm} / \mathrm{sec} 10 \mathrm{~mm} / \mathrm{mV}[0.5-35] \mathrm{Hz} \sim 60 \mathrm{~Hz}$

Figure 6 (A) Standard 12-lead ECG from a patient with Eisenmenger syndrome. Marked right ventricular hypertrophy: $\mathrm{R}>1 \mathrm{mV}$ in $\mathrm{V} 1, \mathrm{R} / \mathrm{S}>1$ in $\mathrm{V} 1, \mathrm{R} / \mathrm{S}<1$ in V4, V5, V6, right atrial hypertrophy, and right axis deviation 153. (B) New electrode placement ECG from the patient shown in figure 6A, QRS axis 155; findings are identical to the standard.

better quality ECGs, with no recalls and without risk for misinterpretations.

- The NEP revealed waveforms that are identical with that of the standard 12-lead ECG, resulting in the same interpretation;

- The new method does not cause disappearance of MI pattern or change $\mathrm{R}$ wave amplitude or QRS axis;
- The NEP was shown suitable and as reliable as the standard for observation of $\mathrm{P}$ waves and arrhythmia diagnosis;

- The NEP can replace standard electrode placements at the wrist and ankles for routine 12-lead electrocardiography.

The NEP is an alternative method to the wrist-ankle placements and has several advantages:

Table 3 Sensitivity and specificity of the NEP method for diagnosis of old inferior MI and normal ECG

\begin{tabular}{|c|c|c|c|c|c|c|}
\hline Diagnosis & & & & & Sensitivity $(95 \% \mathrm{Cl})$ & Specificity (95\% Cl) \\
\hline Old inferior $\mathrm{MI}(\mathrm{n}=1112)$ & & & & & $1.0(0.997$ to 1.0$)$ & $1.0(0.912$ to 1.0$)$ \\
\hline Normal ECG (group C) $(n=276)$ & & & & & $1.0(0.971$ to 1.0$)$ & $1.0(0.976$ to 1.0$)$ \\
\hline Diagnosis & TP & FP & TN & FN & Sensitivity $(95 \% \mathrm{Cl})$ & Specificity $(95 \% \mathrm{CI})$ \\
\hline Old inferior MI $(n=1112)$ & 40 & 0 & 1072 & 0 & $1.0(0.997$ to 1.0$)$ & $1.0(0.912$ to 1.0$)$ \\
\hline Normal ECG (group C) $(n=276)$ & 149 & 0 & 127 & 0 & $1.0(0.971$ to 1.0$)$ & $1.0(0.976$ to 1.0$)$ \\
\hline
\end{tabular}

FN, False negative; FP, False positive; TN, True negative; TP, True positive. 
- Recordings with better quality, no recalls;

- Not having to remove hosiery or other leg garments is convenient and allows more rapid acquisition of ECGs. Time saved is important for therapy, and for hospital and non-hospital laboratories.

The findings have implications worldwide for patients, clinicians, technicians and medical laboratories, and generate the need to study the NEP in patients with probable acute coronary syndrome where timely PCI or thrombolysis can save lives. In addition, the forearms are freed for intravenous or radial artery access and further ECGs that are needed during procedures can be obtained.

Acknowledgements The study could not have enrolled this large number of patients, and completion of Group C could not be accomplished without the help of Lanor Hitch, MLT Manager of Cardiology Services Gamma Dynacare Medical Laboratories. Lanor and her supervisors accomplished the training of technicians for the study. Professor George A Wells, School of Epidemiology, Public Health and Preventative Medicine, University of Ottawa. Director of the Cardiovascular Research Methods Centre, University of Ottawa Heart Institute, and Jordan Bernick MSc provided the statistical analysis. Validation of the study results were completed by an independent interpreter, unknown to the study investigator: Dr Timothy Burns MD, FRCP C, diligently assessed all ECGs from group $C$ patients, along with 40 ECGs showing inferior infarcts, and all shown in the supplement without obtaining remuneration or gifts. $\mathrm{Dr}$ Michele A Turek, Associate Professor, Department of Medicine and Division of Cardiology, University of Ottawa, provided editorial and content support. Stephen Khan assisted with editing, revisions, reformatting of the manuscript, tables and indexing the supplementary material. The author would like to thank Dr Max della Zazzera Medical Director, Bank Medical Centre, for allowing him to use a technician to complete triplicate ECG recordings on patients for the study. Yasmin Khan was responsible for the art work (figure 1). Carrie Barlow assisted with editing of the initial manuscript.

Contributors The Study, design, acquisition, interpretation of data, and writing the manuscript were carried out by GMK who had full access to all of the data in the study and takes responsibility for the integrity of the data and the accuracy of the data analysis.

Competing interests None declared.

Patient consent Obtained.

Ethics approval Gamma-Dynacare Medical Laboratories (GDML).

Provenance and peer review Not commissioned; externally peer reviewed.

Data sharing statement Article Title: A New Electrode Placement Method for obtaining 12-lead ECGs Author: GMK M.D. Email for further information: DrkhanBooks@gmail.com Unpublished Data: 1. ECG artefacts are a major problem in out of hospital laboratories 2. Modified lead placement for Parkinson's disease, tremors, amputees, and patients who must remain seated. 3. Cold extremities and artefacts 4 . How big a problem do we face.

Open Access This is an Open Access article distributed in accordance with the Creative Commons Attribution Non Commercial (CC BY-NC 4.0) license, which permits others to distribute, remix, adapt, build upon this work noncommercially, and license their derivative works on different terms, provided the original work is properly cited and the use is non-commercial. See: http:// creativecommons.org/licenses/by-nc/4.0/

\section{REFERENCES}

1. Wilson FN, Johnson FD, Rosenbaum FF, et al. The precordial electrocardiogram. Am Heart J 1944;27:19-85.

2. Takuma K, Hori S, Sasaki J, et al. An alternative limb lead system for electrocardiographs in emergency patients. Am J Emerg Med 1995;13:514-17.

3. Jowett NI, Turner AM, Cole A, et al. Modified electrode placement must be recorded when performing 12-lead electrocardiograms. Postgrad Med J 2005;81:122-5.

4. Papouchado M, Walker PR, James MA, et al. Fundamental differences between the standard 12-lead electrocardiograph and the modified (Mason-Likar) exercise lead system. Eur Heart $J$ 1987;8:725-33.

5. Mason RE, Likar I. A new system of multiple-lead exercise electrocardiography. Am Heart J 1966;71:196-205.

6. Wilson FN. The distribution of the potential differences produced by the heart beat within the body and its surface. Am Heart $J 1929$ 1930;5:599-616.

7. Einthoven W, Fahr G, de Waart A. On the direction and manifest size of the variations of potential in the human heart and on the influence of the position of the heart on the form of the electrocardiogram. Pflugers Arch 1913;150:275-315, [Translation by: Hoff HE, Sekelj P. Am Heart J 1950;40(2):163-211].

8. Diamond D, Griffith DH, Greenberg ML, et al. Torso mounted electrocardiographic electrodes for routine clinical electrocardiography. J Electrocardiol 1979;12:403-4069.

9. Sevilla DC, Dohrmann ML, Somelofski CA, et al. Invalidation of the resting electrocardiogram obtained via exercise electrode sites as a standard 12-lead recording. Am J Cardiol 1989;63:35-9.

10. Pahlm O, Haisty WK Jr, Edenbrandt L, et al. Evaluation of changes in standard electrocardiographic QRS waveforms recorded from activity-compatible proximal limb lead positions. Am J Cardiol 1992;69:253-7.

11. Kligfield P, Gettes LS, Bailey JJ, American Heart Association Electrocardiography and Arrhythmias Committee, Council on Clinical Cardiology; American College of Cardiology Foundation; Heart Rhythm Society, Josephson M, Mason JW, Okin P, Surawicz B, Wellens $\mathrm{H}$. Recommendations for the standardization and interpretation of the ECG: part I: The electrocardiogram and its technology: a scientific statement from the American Heart Association Electrocardiography and Arrhythmias Committee, Council on Clinical Cardiology; the American College of Cardiology Foundation; and the Heart Rhythm Society: endorsed by the International Society for Computerized Electrocardiology Circulation 2007:115:1306-24 